\title{
Reflector and Protections in a Sodium-cooled Fast Reactor: Modelling and Optimization
}

\author{
David Blanchet ${ }^{1, a}$, Bruno Fontaine ${ }^{1}$ \\ ${ }^{1} C E A, D E N / D E R$, F-13108 Saint-Paul-lez-Durance, France
}

\begin{abstract}
The ASTRID project (Advanced Sodium Technological Reactor for Industrial Demonstration) is a Generation IV nuclear reactor concept under development in France [1]. In this frame, studies are underway to optimize radial reflectors and protections. Considering radial protections made in natural boron carbide, this study is conducted to assess the neutronic performances of the $\mathrm{MgO}$ as the reference choice for reflector material, in comparison with other possible materials including a more conventional stainless steel. The analysis is based upon a simplified 1-D and 2-D deterministic modelling of the reactor, providing simplified interfaces between core, reflector and protections. Such models allow examining detailed reaction rate distributions; they also provide physical insights into local spectral effects occurring at the Core-Reflector and at the Reflector-Protection interfaces.
\end{abstract}

\section{Introduction}

One of the main requirements for radial shields in a pooltype Sodium-cooled Fast Reactor (SFR) is to protect internal vessel structures from radiation damages. The Intermediate Heat Exchanger (IHX) also requires neutron shielding so as to minimize sodium activation in the secondary circuit. The natural choice for neutron absorber in radial shields is the boron carbide, and at a minimum cost, natural boron enrichment offers the longest lifetime. Between core and protections, reflectors can be seen as a transition zone. A good reflector material would preferably have high elastic scattering and low absorption cross sections to enhance the neutron flux level at the core periphery, hence preserving reactivity and flattening fuel burnup distributions. Reflectors are also foreseen to decrease the neutron flux level before protection assemblies in order to preserve their expected lifetime.

The present study is conducted to assess the neutronic performances of an $\mathrm{MgO}$-based reflector material in comparison with a more conventional Stainless Steel reflector. The study also examines other promising candidates. Previous work has already been devoted to characterize the $\mathrm{MgO}$ reflector impact on the outer fuel region [2] and on the minor actinide transmutation in blankets [3]. The present study is revisiting the models and proposes a refined analysis, in particular on reactivity impacts, on power enhancement at the outer fuel region, and on the increased efficiency of the surrounding protections.

\footnotetext{
a Corresponding author: david.blanchet@cea.fr
}

The present study is based on deterministic modelling. Monte-Carlo simulations are only used for punctual validation purposes.

Section $\$ 2$ explains how reflector materials have been selected before investigation, which criteria are considered in the present study. Section $\$ 3$ briefly describes the 1-D cylindrical model and presents the assessment of reflector materials based on this model. The ranking can be done by considering different criteria: based on albedo or on reactivity weights, but also in terms of shielding efficiency when they are associated with protections. Section $\S 4$ is revisiting the 1-D study, by upgrading a $2 \mathrm{D}$ R-Z model. Finally, section $\S 5$ presents some methodological aspects concerning the deterministic modelling and its validation by comparison with Monte-Carlo simulations.

\section{Pre-Selection of Reflector Materials}

The most desirable material characteristics for use in neutron reflectors can be listed below:

- High neutron albedos (ability to reflect neutrons into the core) to preserve reactivity,

- Power enhancement at the core periphery, for radial core power flattening and better fuel utilization,

- High resistance against thermal and mechanical loads (high conductivity, low thermal expansion, low Young modulus), in particular if the material is to be used without cladding,

- High resistance against irradiation damages (acceptable degradation of thermal and mechanical properties),

- Limited swelling under neutron irradiation,

- Low activation for dismantling purpose, 
- Industrial production perspectives,

- Sustainability of the supply chain,

- Acceptable cost (with considerations on the expected lifetime and on the recycling strategy).

Among the large number of potentially good reflector materials, the strategy retained in the present study was to pre-select those having the largest experimental feedbacks, and that could be used with much confidence in the start-up cores of ASTRID. In this regard, previous studies conducted in the past were of interest, like for instance those studies aiming at the development of target matrices for the transmutation of Minor Actinides in Phenix reactor [4] $\left(\mathrm{MgO}, \mathrm{MgAl}_{2} \mathrm{O}_{4}, \mathrm{Y}_{3} \mathrm{Al}_{5} \mathrm{O}_{12}\right)$, but also some prospective studies that were conducted for the development of the Gas-cooled Fast Reactors $\left(\mathrm{Zr}_{3} \mathrm{Si}_{2}\right.$, $\mathrm{SiC}$ ) [5] or associated with fusion Reactors technologies [6] (V-4Ti-4Cr, Beryllium). Hydride materials, like $\mathrm{CaH}$ or $\mathrm{ZrH}$ (having a strong moderation impact), were directly disqualified from this pre-selection because of safety considerations (hydrogen risks in accidental conditions).

Hence, the resulting short list of reflector materials to be further examined in the present neutronic study are: Magnesium Oxide $(\mathrm{MgO})$, Spinel $\left(\mathrm{MgAl}_{2} \mathrm{O}_{4}\right)$, Yttrium Aluminium Garnet $\left(\mathrm{Y}_{3} \mathrm{Al}_{5} \mathrm{O}_{12}\right)$, Zirconium Silicide $\left(\mathrm{Zr}_{3} \mathrm{Si}_{2}\right)$, Silicium Carbide (SiC), Zirconium Carbide (ZrC), Titanium Carbide (TiC), Beryllium (Be), Vanadium alloy (V-4Ti-4Cr), depleted Uranium Oxide $\left(\mathrm{UO}_{2}\right)$ as a fertile material, and ferritic martensitic stainless steel (denoted as "EM10"). These pre-selected materials are examined in a comparative study where the reference case is a standard reflector made of a stainless steel ("EM10"). The use of fertile material being excluded on grounds of proliferation concerns, it is considered here for exercise only.

\section{A Simplified One-Dimensional Model for Material Assessment}

\subsection{Model Description}

A simple one-dimensional cylindrical reactor model is setup with the aim of being almost representative of the ASTRID core. The geometry illustrated in Figure 1 is a radial cross-cut of the reactor model, comprising the fuel zone, the reflector zone (denoted as "R") and the radial protections or shielding (denoted as "P"), the rest of the geometry being filled with sodium from $350 \mathrm{~cm}$ up to $600 \mathrm{~cm}$.

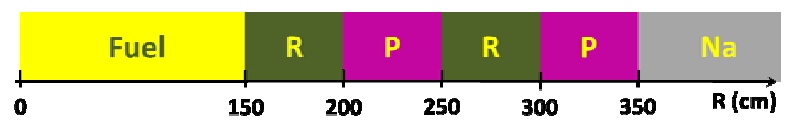

Figure 1. 1-D cylindrical reactor model.

Neutron transport simulations are based on the deterministic code system ERANOS (version 2.4) [4] and the Monte-Carlo code TRIPOLI-4® (version 9) [5]. Both codes are used in conjunction with the JEFF3.1 nuclear data library [6].
The Sn code BISTRO is used with a S4P1 angular quadrature and a spatial scheme based on finite differences. Two calculation routes are being considered: one is a "project route" based on a broad energy mesh in 33 groups; the other is a "reference route" based on a fine energy mesh in 1968 groups. For the rest of the study, they will be respectively denoted as "E2.33g" and "E2.1968g". The neutron cross sections are self-shielded (using the subgroup method) and homogenized at the sub-assembly level by the lattice code ECCO. "E2.33g" only differs from "E2.1968g" by the extra step of condensation in energy.

For simplicity reasons and to avoid any ambiguities when comparing with Monte-Carlo results, calculations are performed at room temperature $\left(20^{\circ} \mathrm{C}\right)$. The geometry is discretized into mesh sizes of $1 \mathrm{~cm}$, with "specular reflection" boundary conditions at $\mathrm{R}=0$ and "zero flux" boundary conditions at $\mathrm{R}=600 \mathrm{~cm}$. This simplified model is representative of radial flux gradients and local distributions at the various interfaces, but it is quite unrealistic for reactivity analysis. Indeed, the specular reflections imposed in axial directions are convenient for comparisons with Monte-Carlo simulations in which no leakage model is implemented, but the artificially high reactivity level induced by these conditions is preventing any comparative analysis on the reactivity-weight of reflectors. In deterministic modelling however, an axial buckling value can be easily derived to find a critical state, much more suitable for reactivity analysis. A second deterministic model is hence dedicated to reactivity analysis.

\subsection{Material Ranking by Albedo}

A priori, the best indicator for the assessment of reflector materials is a dimensionless quantity called the "albedo" denoted $\gamma$ and defined as the ratio of the neutron flux reflected (or back scattered) by the material over the neutron flux entering the material:

$$
\gamma=\frac{\mathrm{J}-}{\mathrm{J}+}
$$

In the above equation, $\mathrm{J}$ stands for the neutron current at the interface between core and reflector, J- denoting the neutrons back-scattered into the core and $\mathrm{J}+$ representing the neutron leakage from the core. Based on the 1-D model presented in section $\S 3.1$, Table 1 gives comparative results of albedos calculated for the different reflector materials.

Table 1. Reflector material ranking by albedo.

\begin{tabular}{|c|c|c|}
\hline Rank & Material & Albedo \\
\hline 1 & $\mathbf{M g O}$ & 0,859 \\
\hline 2 & $\mathbf{M g A l}_{\mathbf{2}} \mathbf{O}_{4}$ & 0,853 \\
\hline 3 & $\mathbf{Y}_{\mathbf{3}} \mathbf{A l}_{\mathbf{5}} \mathbf{O}_{\mathbf{1 2}}$ & 0,847 \\
\hline 4 & $\mathbf{Z r}_{\mathbf{3}} \mathbf{S i}_{2}$ & 0,833 \\
\hline 5 & $\mathbf{S i C}$ & 0,831 \\
\hline
\end{tabular}




\begin{tabular}{|c|c|c|}
6 & ZrC & 0,827 \\
\hline 7 & TiC & 0,823 \\
\hline 8 & $\mathbf{V - 4 T i - 4 C r}$ & 0,821 \\
\hline 9 & $\mathbf{B e}$ & 0,807 \\
\hline 10 & $\mathbf{E M 1 0}$ & 0,802 \\
\hline 11 & $\mathbf{U O}_{2}$ & 0,744 \\
\hline
\end{tabular}

According to Table 1, the ranking in terms of "neutron reflection capabilities" is indicated by decreasing albedos: $\mathrm{MgO}>\mathrm{MgAl}_{2} \mathrm{O}_{4}>\mathrm{Y}_{3} \mathrm{Al}_{5} \mathrm{O}_{12}>$ $\mathrm{Zr}_{3} \mathrm{Si}_{2}>\mathrm{SiC}>\mathrm{ZrC}>\mathrm{TiC}>\mathrm{V}-4 \mathrm{Ti}-4 \mathrm{Cr}>\mathrm{Be}>\mathrm{EM} 10>$ $\mathrm{UO}_{2}$.

\subsection{Material Ranking by Reactivity weight}

Another indicator providing a ranking of best reflector materials is the reactivity of the system. Reactivity results are given in Table 2 .

Table 2. Reflector material ranking by reactivity.

\begin{tabular}{|c|c|c|}
\hline Rank & Material & $\begin{array}{c}\text { Reactivity } \\
\text { (pcm) }\end{array}$ \\
\hline 1 & $\mathbf{M g O}$ & 30618 \\
\hline 2 & $\mathbf{M g A l}_{\mathbf{2}} \mathbf{O}_{\mathbf{4}}$ & 30607 \\
\hline 3 & $\mathbf{Y}_{\mathbf{3}} \mathbf{A l}_{\mathbf{5}} \mathbf{O}_{\mathbf{1 2}}$ & 30596 \\
\hline 4 & $\mathbf{Z r}_{\mathbf{3}} \mathbf{S i}_{\mathbf{2}}$ & 30579 \\
\hline 5 & $\mathbf{V - 4 T i - 4 C r}$ & 30556 \\
\hline 6 & $\mathbf{S i C}$ & 30553 \\
\hline 7 & $\mathbf{Z r C}$ & 30550 \\
\hline 8 & $\mathbf{T i C}$ & 30532 \\
\hline 9 & $\mathbf{E M 1 0}$ & 30517 \\
\hline 10 & $\mathbf{B e}$ & 30494 \\
\hline 11 & $\mathbf{U O 2}$ & 30413 \\
\hline
\end{tabular}

In the absence of axial leakage, such a 1-D model is only representative of the radial neutron leakages, and thereby exhibits an artificially very high reactivity level (because of neutron reflections). These reactivities are of course quite unrealistic and the differences found from one material to another are far from being representative of the results that could be obtained in a real 3D modelling with correct leakages and considering burnup distributions. However, it gives a fair idea of the potential ranking, since it almost confirms all the previous results based on albedos.

\subsection{Material Ranking by Shielding Efficiency}

The choice of a reflector material can also be guided by its efficiency in terms of neutron shielding when it is associated with radial protections made of neutron absorber. Let us give a fixed design for the radial protections in order to examine solely the impact of the reflector materials on the efficiency of the reflector - protection system. The indicator is the neutron flux attenuation. Neutron fluxes are calculated at $\mathrm{R}=5 \mathrm{~m}$ from core center, which is the approximate location of Intermediate Heat Exchangers (IHX). The aim is not to obtain absolute values but to understand the trends. Neutron flux results are summarized in Table 3.

Table 3. Reflector material ranking by shielding efficiency.

\begin{tabular}{|c|c|c|}
\hline Rank & Material & $\begin{array}{c}\Phi \text { at IHX } \\
\left(\mathbf{n . c m}^{-2} \cdot \mathbf{s}^{-1}\right)\end{array}$ \\
\hline 1 & $\mathbf{B e}$ & 126 \\
\hline 2 & $\mathbf{T i C}$ & 449 \\
\hline 3 & $\mathbf{Z r C}$ & 618 \\
\hline 4 & $\mathbf{M g O}$ & 1801 \\
\hline 5 & $\mathbf{U O}_{2}$ & 2161 \\
\hline 6 & $\mathbf{E M 1 0}_{2}$ & 2385 \\
\hline 7 & $\mathbf{M g A l}_{2} \mathbf{O}_{4}$ & 3095 \\
\hline 8 & $\mathbf{S i C}_{4}$ & 3562 \\
\hline 9 & $\mathbf{Y}_{\mathbf{3}} \mathbf{A l}_{\mathbf{5}} \mathbf{O}_{\mathbf{1}}$ & 4483 \\
\hline 10 & $\mathbf{V}_{\mathbf{4}} \mathbf{T T i}_{\mathbf{4}} \mathbf{4} \mathbf{r}$ & 5496 \\
\hline 11 & $\mathbf{Z r}_{\mathbf{3}} \mathbf{S i}_{2}$ & 8997 \\
\hline
\end{tabular}

According to Table 3, the best material ranking is: $\mathrm{Be}$ $>\mathrm{TiC}>\mathrm{ZrC}>\mathrm{MgO}>\mathrm{UO}_{2}>\mathrm{EM} 10>\mathrm{MgAl}_{2} \mathrm{O}_{4}>\mathrm{SiC}>$ $\mathrm{Y}_{3} \mathrm{Al}_{5} \mathrm{O}_{12}>\mathrm{V}-4 \mathrm{Ti}-4 \mathrm{Cr}>\mathrm{Zr}_{3} \mathrm{Si}_{2}$. This ranking is directly correlated with the moderation efficiency of the reflector material, since neutron moderation is improving the efficiency of the neutron absorbers in radial protections. These results are illustrated below in Figure 2.

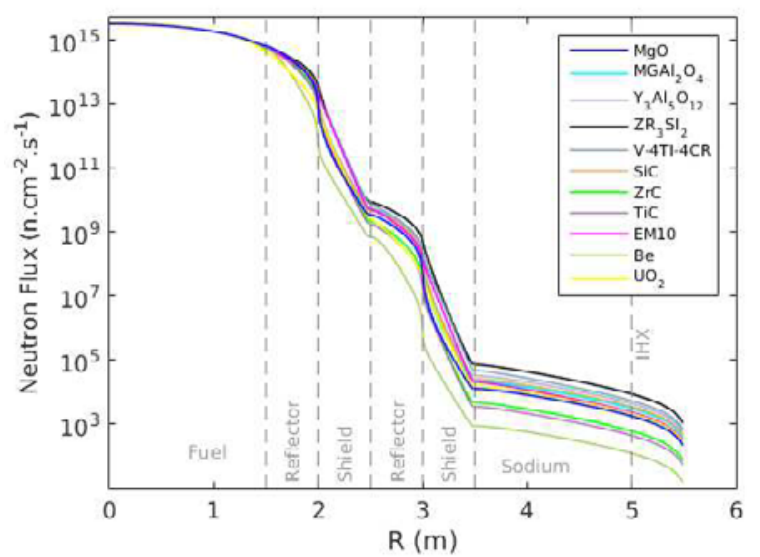

Figure 2. Neutron flux attenuation through the core-reflectorprotection system.

One can however put in question the pertinence of such an indicator since the axial leakages are largely predominant in the ASTRID core concept. The next section is an attempt to address this problem by considering a 2D RZ modelling.

\section{Considerations on Axial Leakage}


This section examines a 2D-RZ modelling of the ASTRID reactor. This simple reactor model is presented in Figure 3 below.

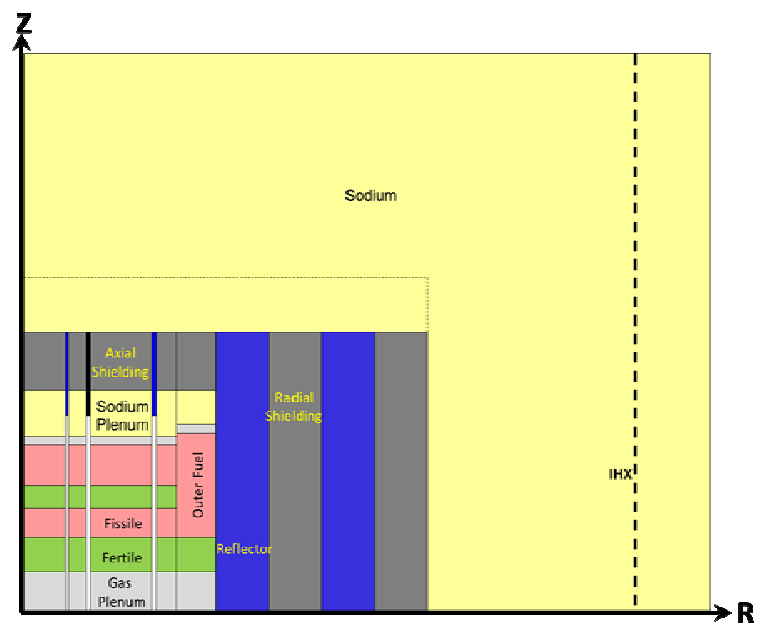

Figure 3. 2D-RZ model of the ASTRID reactor.

In a preliminary approach, most of design details concerning the core surroundings are voluntary ignored because information is lacking and there are still some unknowns in the specifications. For instance, the IHX is still in a design phase, with possible change in axial position, with regular updates in geometry or shielding options. Hence, the IHX is only represented here by its expected position (see the dashed line in Figure 3). Similarly, all vessel internals, sub-assembly head structures are simplified and represented by homogeneous sodium. As a consequence, the present model will not serve in determining absolute values, but will rather be useful to investigate shielding options in design studies, and to understand the main contributions.

Reactivity results are given in Table 4 . These results are clearly confirming the trends already indicated by the one-dimensional study presented in $\$ 3.3$.

Table 4. Reflector material ranking by reactivity (2D-RZ).

\begin{tabular}{|c|c|c|}
\hline Rank & Material & Reactivity \\
\hline- & - & pcm \\
\hline 1 & $\mathbf{M g O}$ & 7963 \\
\hline 2 & $\mathbf{M g A l}_{\mathbf{2}} \mathbf{O}_{\mathbf{4}}$ & 7829 \\
\hline 3 & $\mathbf{Y}_{\mathbf{3}} \mathbf{A l}_{\mathbf{5}} \mathbf{O}_{\mathbf{1 2}}$ & 7707 \\
\hline 4 & $\mathbf{B e}$ & 7526 \\
\hline 5 & $\mathbf{S i C}$ & 7514 \\
\hline 6 & $\mathbf{Z r C}$ & 7471 \\
\hline 7 & $\mathbf{T i C}$ & 7445 \\
\hline 8 & $\mathbf{Z r}_{\mathbf{3}} \mathbf{S i} \mathbf{2}_{\mathbf{2}}$ & 7408 \\
\hline 9 & $\mathbf{V - 4 T i - 4 C r}_{\mathbf{4}}$ & 7362 \\
\hline 10 & $\mathbf{E M 1 0}_{\mathbf{1}}$ & 7139 \\
\hline 11 & $\mathbf{U O}_{\mathbf{2}}$ & 6645 \\
\hline
\end{tabular}

The neutron flux is calculated and plotted in Figure 4.

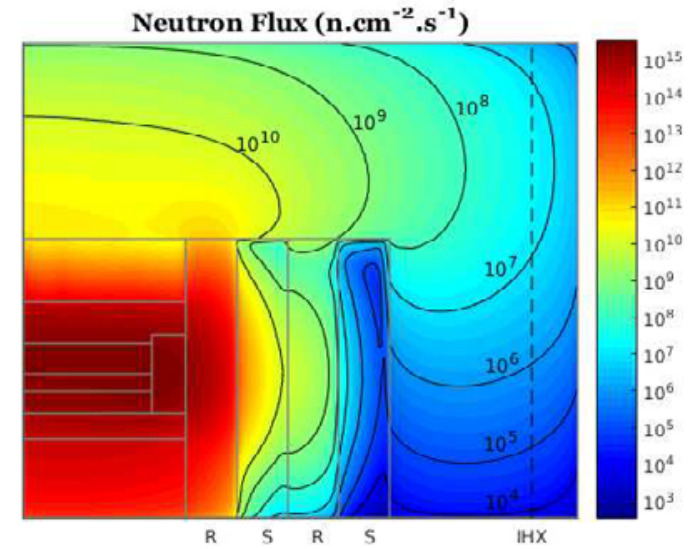

Figure 4. A $2 \mathrm{D}-\mathrm{RZ}$ neutron flux map, illustrating the neutron attenuation in radial direction through the core-reflectorprotection system, and the counterbalance by neutron bypass with axial leakages.

This illustration highlights a major contribution from the axial neutron leakages when determining the secondary sodium activation. According to this Figure, radial shielding looks already satisfactory and there is no need to further improve the efficiency of the radial reflectorprotection systems. However, if one wants to decrease the level of secondary sodium activation in the IHX, most of the efforts should be devoted to develop better shielding against axial neutron leakages.

The heterogeneous axial arrangement of the ASTRID core is designed to minimize the sodium void reactivity effect during accident scenarios. This layout also results in significant axial neutron leakages under normal operation. Even though the upper shielding contains strong neutron absorbers, with highly enriched Boron carbide (up to $90 \%{ }^{10} \mathrm{~B}$ content) in the lower part and naturally enriched boron carbide in the upper part, the axial length available for protections is rather limited by severe constraints on total core height. In comparison, there is much space available for radial protections. In conclusion nothing serves to further optimize radial protections if neutrons are bypassing through axial leakages.

\section{Validation studies by Comparison with Monte-Carlo Simulations}

The validation studies presented in this section are restricted to the $\mathrm{MgO}$ reflector and to the more conventional Stainless Steel reflector. These studies were conducted to investigate local reaction rates at the interfaces (e.g. core-reflector), and also shielding aspects. Recommendations are issued for the improvement of deterministic modelling on these aspects.

\subsection{Local interfaces}

The core-reflector and the reflector-protection interfaces are very challenging for deterministic neutron transport modelling. The reflector is a scattering zone in sandwich between the fuel and the protections, which are two highly absorbing zones. 
The $\mathrm{MgO}$ reflector material produces a slightly softer neutron spectrum than the stainless steel. Neutron slowing down is indeed favoured by lightest elements. This softening of the neutron spectrum has consequences on both sides: on the fuel side where the $\mathrm{MgO}$ is reflecting $20 \%$ more neutrons into the core than the stainless steel and on the protection side where the shielding is enhanced. The strength of the neutron flux attenuation can be observed with a log-scale in figure 5 .

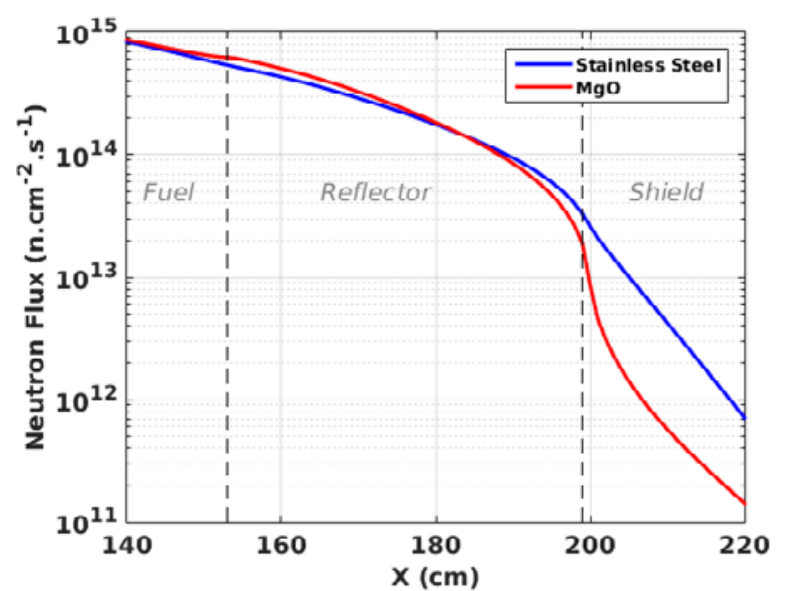

Figure 5. Neutron flux attenuation through the core-reflectorprotection system, comparison between $\mathrm{MgO}$ and stainless steel reflectors.

The capture rate distributions are plotted in figure 6 and figure 7 for each of the two reflectors under study. The local spectral effects previously mentioned are increasing the capture rates, with characteristic peaking at the interfaces. In comparison to reference Monte-Carlo simulations, the project route calculations (defined in $\S 3.1)$ are significantly overestimating the efficiency of the protections $(+10 \%$ and $+17 \%$ in capture rates at the reflector-protection interface respectively for stainless steel and $\mathrm{MgO}$ ). The accurate prediction of these local effects would require better modelling of the cross sections; some improvements are suggested in the process of homogenizing neutron cross sections, both in radial protections and in fuel regions close to the reflectors.

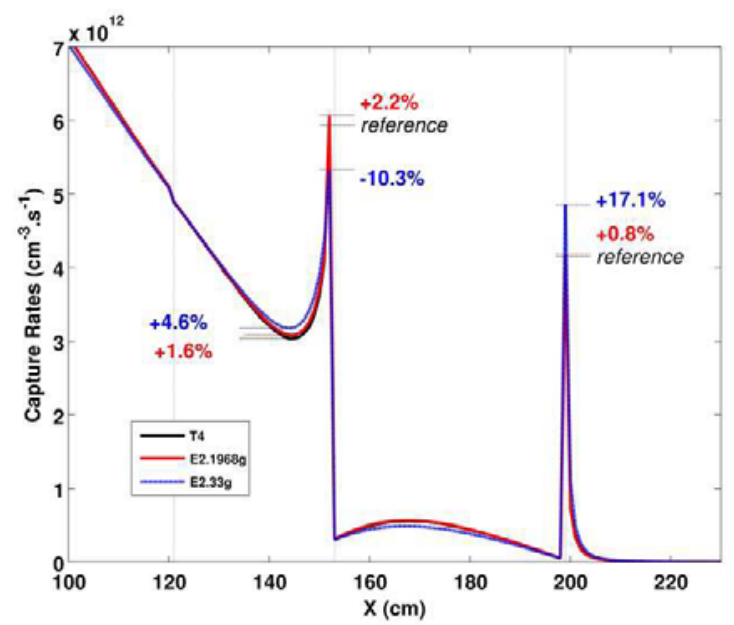

Figure 6. Capture rate distributions in the case of $\mathrm{MgO}$ reflector.

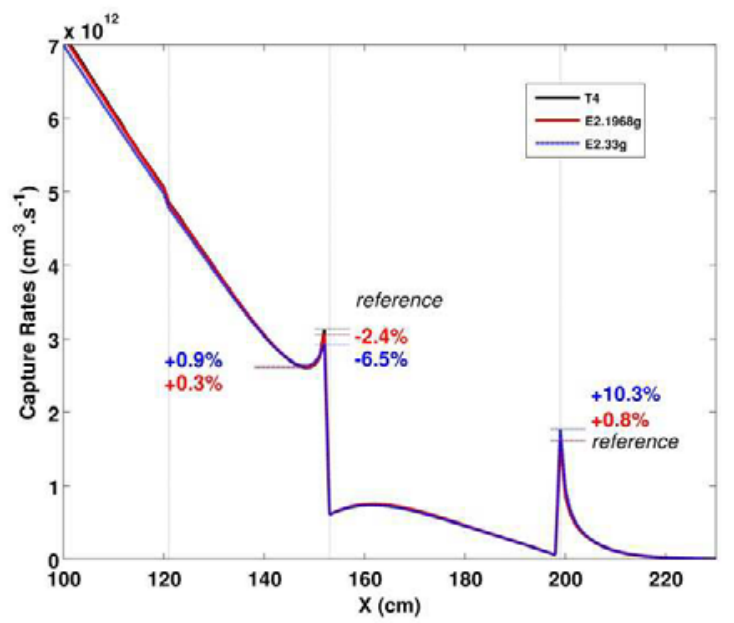

Figure 7. Capture rate distributions in the case of Stainless Steel reflector.

\subsection{Shielding aspects}

Monte-Carlo simulations are widely used in radiation shielding problems for the following reasons: it is easyto-model any complex geometry, there is no discretization in energy/angle variables, the resolution method is naturally adapted to parallel machines and there are increasing computing resources available. Moreover, in the particular case of shielding calculations, for deep penetration problems where radiations are attenuated by several decades, or in bypass problems where very rare events are questioning the statistical significance of the results, some biasing games can be done. The variance reduction approach using biasing techniques however requires some verification because it introduces some deterministic modelling assumptions. These assumptions and the verification steps associated are briefly recalled hereafter for the specific case of the present study.

In a first step, analog simulations (i.e. simulations not using variance reduction, with the exception of implicit captures treatment...) are launched in critical mode. The fission source distributions are calculated. In a second step, a source calculation propagates the fission source distributions obtained at the previous step. Results obtained in step 1 and 2 are verified to be in very close agreement. Once verified, the source calculation can be improved in step 3, by using variance reduction technique. The biasing technique used in the TRIPOLI$4{ }^{\circledR}$ code (with the INIPOND module) is based on the automatic generation of an importance map [10] [11]. The biasing energy mesh chosen for the present study is based on a 6 group structure defined by the following boundaries: $20,4,1,10^{-2}, 10^{-4}, 10^{-6}, 10^{-11} \mathrm{MeV}$. The spatial mesh used for the ponderation is covering the whole cylindrical geometry of the reactor by ultra-thin steps of $1 \mathrm{~cm}$ length.

Step 1 and step 3 are simulated with $10^{9}$ particles. The results are plotted together with the corresponding 
ERANOS-2 results in Figure 8 and Figure 9 for the two cases of reflector. The variance reduction technique works perfectly to push neutrons through the reflectors and the protection rings. The very small statistical errors obtained in neutron flux close to the detector (at $\mathrm{R}=500$ $\mathrm{cm}$ ) indicate a very good convergence of the simulations using the variance reduction technique; while natural simulations do not provide any result at all at this location.

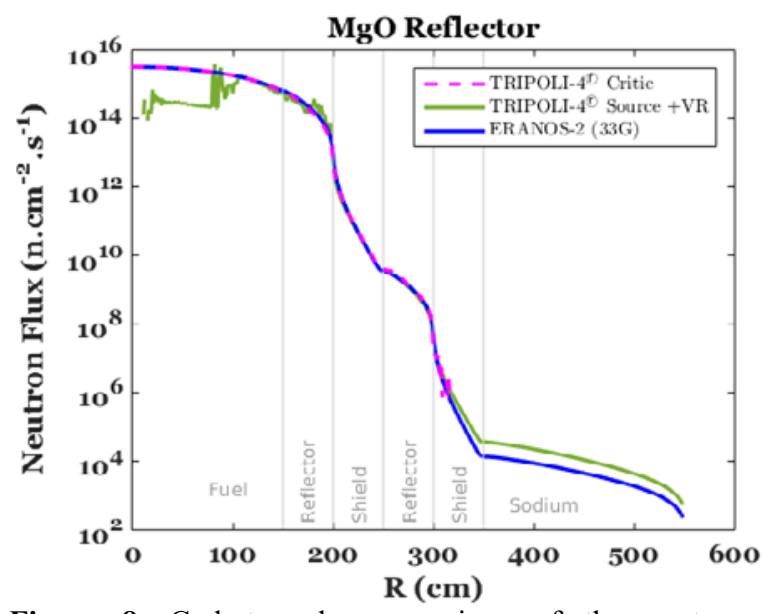

Figure 8. Code-to-code comparison of the neutron flux attenuation in the case of $\mathrm{MgO}$ reflector.

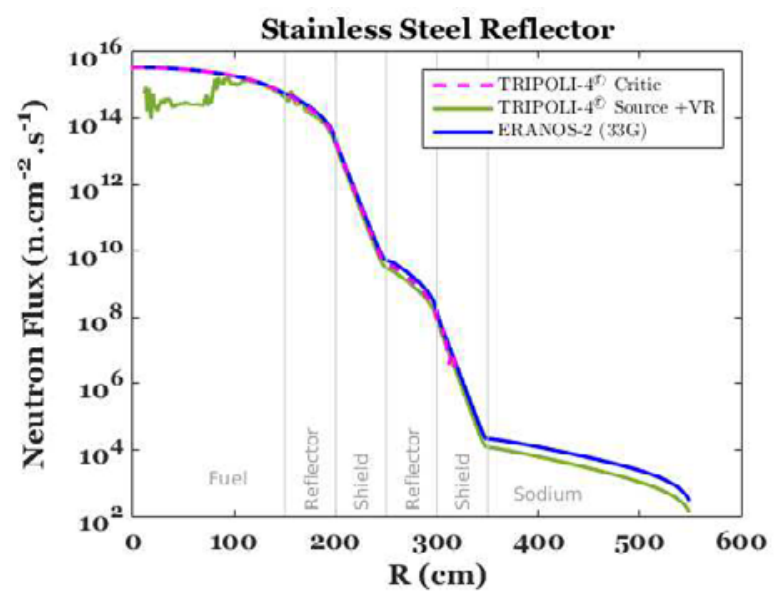

Figure 9. Code-to-code comparison of the neutron flux attenuation in the case of EM10 reflector.

The ERANOS-2 and TRIPOLI-4® neutron flux results are compared in terms of ratios in Table 5. Since we are dealing with simplified models, the absolute flux values are not of interest here, so only ratios are reported in Table 5. At $\mathrm{R}=5 \mathrm{~m}$, after 12 decades of attenuation, the neutron flux ratios are $\sim 0.4$ and $\sim 1.9$ respectively for the $\mathrm{MgO}$ and stainless steel reflectors.

Table 5. Ratio of ERANOS- 2 and TRIPOLI-4® neutron flux results obtained at IHX location $(\mathrm{R}=5 \mathrm{~m})$.

\begin{tabular}{|l|c|}
\hline & $\Phi \mathrm{E} 2 / \Phi \mathrm{T} 4( \pm 1 \sigma)$ \\
\hline $\mathrm{MgO}$ & $0.38( \pm 0.7 \%)$ \\
\hline Stainless Steel & $1.85( \pm 2.7 \%)$ \\
\hline
\end{tabular}

This agreement seems quite reasonable for the purpose of doing parametric studies in a pre-design phase.

\section{Conclusion}

The Magnesium Oxide material is the reference choice for the ASTRID reflector. It has the required properties for a good reflector: radiation resistance and stability at high temperatures, low absorption and high scattering cross sections... It also benefits from a large experimental feedback since it was irradiated in the Phenix SFR as target matrix in the frame of Minor Actinide transmutation studies. The aim of the present study was to verify this choice of reflector material by comparing its neutronic performances with other material candidates, including a more conventional stainless steel reflector. The analysis is based upon the use of simplified 1-D and 2-D cylindrical models characterizing well the interfaces of interest: i.e. the core-reflector and the reflector-protection. The criteria retained for the ranking of reflector materials are purely based on neutronic considerations: reactivity of core-reflector systems, averaged albedo at core-reflector interfaces and, in association with protections, the efficiency in neutron attenuation, so as to minimize secondary sodium activation through the intermediate heat exchanger. The comparisons are clearly in favour of the $\mathrm{MgO}$ reflector, even though $\mathrm{MgAl}_{2} \mathrm{O}_{4}$ or $\mathrm{Y}_{3} \mathrm{Al}_{5} \mathrm{O}_{12}$ are very close in terms of reactivity, and $\mathrm{TiC}$ or $\mathrm{ZrC}$ are slightly better in shielding. The flattening in radial core power distribution, which is important for better fuel utilization in the outer core region, was not explicitly considered as ranking criteria (but it is redundant with the reactivity one); however this aspect was investigated in details in [2].

Monte-Carlo simulations were conducted only for validation purposes, especially on local reaction rates at the interfaces (e.g. core-reflector), and also on shielding aspects. The deterministic results obtained with ERANOS-2 code system were found in reasonable agreement with the reference Monte-Carlo simulations using TRIPOLI-4®. Some improvements are suggested in the process of multi-group cross-section generation.

There is still a strong interest in developing 3D deterministic modelling, especially for design studies, since it provides useful indications for understanding trends and getting sensitivities; moreover, it allows propagation of uncertainties. This study is relying intentionally on very simplified reactor models (1-D and 2-D cylindrical) using deterministic codes. This approach is relevant for the design phase, because a lot of design specifications are still unknown or might be changing. For instance, the description of internal vessel structures, the position and geometry of the intermediate heat exchanger will have a direct impact on the determination of the secondary sodium activation. As long as the reactor description is incomplete, there is limited interest in determining only absolute values, because some of the most important modelling details could be forgotten.

\section{References}

1. J. Rouault, "The ASTRID Project: Status and Prospects at the Beginning of the Basic Design 
Phase", in proceedings of ICAPP 2016, San Francisco, USA, 2016.

2. D. Blanchet, B. Fontaine, "On Core-Reflector Interface Effects in the ASTRID Sodium-Cooled Fast Reactor", in proceedings of ICAPP 2016, San Francisco, USA, 2016.

3. L. Buiron, B. Fontaine, "SFR Minor Actinide Bearing Blanket Fine Characterization Using MonteCarlo", in proceedings of ICAPP 2016, San Francisco, USA, 2016.

4. J.M. Bonnerot et al., "Progress on inert Matrix Fuels for Minor Actinides Transmutation in Fast Reactor", in Proceedings of GLOBAL 2007, Bise, Idaho, USA, 2007.

5. M. Le Flem, J. Canel and S. Urvoy, "Processing and Characterization of $\mathrm{Zr}_{3} \mathrm{Si}_{2}$ for Nuclear Applications", Journal of Alloys and Compounds 465, 269-273, 2008.

6. D.L. Smith et al., "Development of Vanadium-Base Alloys for Fusion First-Wall-Blanket Applications", Fusion Engineering and Design 29, 388-410, 1995.

7. J.M. Ruggieri, J.F. Lebrat, J. Tommasi, P. Archier, "Status of ERANOS-2 code system validation for Sodium Fast Reactor Applications", in proceedings of FR09, Kyoto, Japan, 2009.

8. E. Brun, F. Damian, C.M. Diop, E. Dumonteil, F.-X. Hugo, C. Jouanne, "TRIPOLI-4®, CEA, EDF and AREVA reference Monte Carlo code", in Proceedings of SNA+MC2013, Paris, France, 2013.

9. A. Santamarina et al., "The JEFF-3.1.1 nuclear data library, JEFF report 22, validation results from JEF2.2 to JEFF-3.1.1”, vol. 6807. OECD, NEA, 2009.

10. J.-P. Both, J.-C. Nimal, T. Vergnaud, “Automated Importance Generation and Biasing Techniques for Monte-Carlo Shielding Techniques by the TRIPOLI3 Code", Progress in Nuclear Energy, Vol 24, pp.273-281, 1990.

11. O. Petit, Y.-K. Lee, C.M. Diop, "Variance Reduction Adjustment in Monte-Carlo TRIPOLI-4® Neutron Gamma Coupled Calculations", Progress in Nuclear Science and Technology, Vol 4, pp.408-412, 2014.

12. N. Chapoutier, A. Janet, G. Nolin, M.-C. Ricol, A.C. Scholer, D. Verrier, Ch. Venard, "ASTRID Core Shielding - Design Studies and Benchmark Analysis", in proceedings of ICAPP 2015, Nice, France, 2015. 